\title{
GROUPOIDS AND COMPACT QUANTUM GROUPS
}

\author{
ALBERT J. L. SHEU \\ Department of Mathematics, University of Kansas \\ Lawrence, KS 66045, USA \\ E-mail: sheu@kuhub.cc.ukans.edu
}

1. Introduction. In this article, we explain how compact quantum groups are related to groupoids.

It is remarkable that in the (algebraic) theory of quantum groups developed by Drinfeld and many others [D, RTF], one gets a deformation of both the Poisson structure and the group structure simultaneously, for a multiplicative Poisson structure on a Lie group. Further studies showed that [So1] the algebra of compact quantum groups is closely related to the underlying singular symplectic foliation [We, LuWe] and that Rieffel's deformation quantization [Ri1, Ri2, Ri3] in this context exhibits very subtle properties [Ri4, Sh2, Sh3, Sh4] with regard to the symplectic foliation.

Parallel to the algebraic theory of quantum groups, Woronowicz initiated an analytical ( $\mathrm{C}^{*}$-algebraic) theory of quantum groups [Wo1, Wo2] which successfully provides a general $\mathrm{C}^{*}$-algebraic framework for compact quantum groups. Since Connes' successful use $[\mathrm{Co}]$ of groupoid $\mathrm{C}^{*}$-algebras $[\mathrm{Re}]$ in the study of foliations, it has been well recognized that groupoid $\mathrm{C}^{*}$-algebras provide a useful tool in studying operator algebras [CuM, MRe, Sh1] (which often arise from geometric objects). We found that Vaksman and Soibelman's result [So1, VSo2] relating the singular symplectic foliation to representations of the algebra of compact quantum groups and quantum spheres can be used to establish a relation between these quantum spaces and some fundamental 'discrete' groupoids [Sh5]. In fact, the algebra of such a quantum space forms the 'core' of the groupoid $\mathrm{C}^{*}$-algebra of a 'discrete' groupoid, and for quantum spheres and quantum $S U(3)$, it is actually equal to some groupoid $\mathrm{C}^{*}$-algebras.

2. Compact matrix quantum groups. In this section, we follow the setting used in [LeSo] to summarize the results about compact matrix quantum groups that we need

1991 Mathematics Subject Classification: Primary 17B37; Secondary 22A22, 22D25, 46L60, 46L87, 58F05, 81R50, 81S10.

The paper is in final form and no version of it will be published elsewhere. 
in establishing a connection with groupoid.

For a simple complex Lie group $G$, we fix a root system $\Lambda$ with (positive) simple roots $\left\{\alpha_{i}\right\}_{i=1}^{r}$ for its Lie algebra $\mathfrak{g}$. (Here we use $\Lambda$ for the root system instead of $\Delta$ which will be used for the comultiplication.) There corresponds a Cartan-Weyl basis $\left\{X_{\alpha}\right\}_{\alpha \in \Lambda} \cup\left\{H_{i}\right\}_{i=1}^{r}$ with $H_{i}=\left[X_{\alpha_{i}}, X_{-\alpha_{i}}\right]$ for each $i$. Let $\mathfrak{k}$ be the real form (i.e. the +1 -eigenspace) for the antilinear involution $\omega: \mathfrak{g} \rightarrow \mathfrak{g}$ defined by $\omega\left(X_{\alpha}\right)=-X_{-\alpha}$ and $\omega\left(H_{i}\right)=-H_{i}$ for all $\alpha \in \Lambda$ and $1 \leq i \leq r$. It is easy to see that $\mathfrak{k}$ is the $\mathbb{R}$-linear span of $X_{\alpha}-X_{-\alpha}, i X_{\alpha}+i X_{-\alpha}$, and $i H_{i}$ in $\mathfrak{g}$. It is well-known that $\mathfrak{k}$ is the Lie algebra of a maximal compact subgroup $K$ of $G$. The pair $(G, K)=(S L(n, \mathbb{C}), S U(n))$ is a fundamental example.

It is known [B-D, So2] that all multiplicative Poisson structures on $G$ and on $K$ are determined (up to an isomorphism) by $p=a \mathbf{r}+v$ with $a \in \mathbb{R}$ and $v \in \mathfrak{h} \wedge \mathfrak{h}$, where

$$
\mathbf{r}=\frac{i}{2} \sum_{\alpha \in \Lambda_{+}}\left(X_{-\alpha} \otimes X_{\alpha}-X_{\alpha} \otimes X_{-\alpha}\right) \in \mathfrak{k} \wedge \mathfrak{k}
$$

and $\mathfrak{h}$ is the real Cartan subalgebra linearly spanned by $H_{i}$ 's over $\mathbb{R}$. In fact, $\pi_{x}:=$ $L_{x} p-R_{x} p$ gives the Poisson 2-tensor [We] at $x \in K$ where $L_{x}$ and $R_{x}$ are the left and right translations by $x$.

Each fixed $p$ determines a family of quantum groups ${ }_{p} K_{q}, q \geq 1$, (or more precisely, a family of Hopf *-algebras $C\left({ }_{p} K_{q}\right)^{\infty}$ of regular functions on $\left.{ }_{p} K_{q}\right)$ which deforms the Poisson structure corresponding to $p$. In the following, we shall concentrate on the standard case of $K_{q}={ }_{\mathbf{r}} K_{q}$ with $p=\mathbf{r}$.

By classifying all irreducible ${ }^{*}$-representations of $C\left(K_{q}\right)^{\infty}$ on Hilbert spaces, Soibelman completed $C\left(K_{q}\right)^{\infty}$ into a type-I $\mathrm{C}^{*}$-algebra $C\left(K_{q}\right)$. On the other hand, starting from a purely $\mathrm{C}^{*}$-algebraic setting, Woronowicz developed a general framework for $\mathrm{C}^{*}$ algebraic (compact) quantum groups [Wo2] and proved the existence of the important invariant Haar functional $h$ which will be discussed later.

Recall that [Wo1, VSo1] the $\mathrm{C}^{*}$-algebra $C\left(S U(2)_{q}\right)$ is generated by $u_{i j}$, with $1 \leq i, j \leq$ 2 , satisfying $u_{22}=u_{11}^{*}, u_{12}=-q^{-1} u_{21}^{*}$, and $u^{*} u=u u^{*}=1$. An important irreducible (non-faithful) *-representation $\pi_{0}$ of $C\left(S U(2)_{q}\right), q>1$, on $\ell^{2}\left(\mathbb{Z}_{\geq}\right)$is given by

$$
\pi_{0}(u)=\left(\begin{array}{cc}
\alpha & -q^{-1} \gamma \\
\gamma & \alpha^{*}
\end{array}\right)
$$

where $\alpha\left(e_{j}\right)=\left(1-q^{-2 j}\right)^{1 / 2} e_{j-1}$ and $\gamma\left(e_{j}\right)=q^{-j} e_{j}$ for $j \geq 0$. Here $\pi_{0}$ is applied to $u=\left(u_{i j}\right)$ entrywise.

The well-known canonical embedding $\phi_{i_{*}}: S U(2) \rightarrow K$ for the basic triple

$$
\left\{X_{\alpha_{i}}, X_{-\alpha_{i}}, H_{i}\right\}, \quad 1 \leq i \leq r,
$$

induces a Hopf *-algebra homomorphism $\phi_{i}: C\left(K_{q}\right)^{\infty} \rightarrow C\left(S U(2)_{q}\right)^{\infty}$. We call $\pi_{i}:=$ $\pi_{0} \circ \phi_{i}$ the fundamental representations of $C\left(K_{q}\right)^{\infty}$.

Recall that the Weyl group $W$ of $K$ is a Coxeter group (c.f. [H] and the reference there for details) generated by $\left\{s_{i}\right\}_{i=1}^{r}$ with $\left(s_{i} s_{j}\right)^{m_{i j}}=1$ for $m_{i i}=1$ and some $m_{i j} \in\{2,3,4,6\}$ if $i \neq j$, where $s_{i}=s_{\alpha_{i}}$ is the reflection on $\mathfrak{h}^{*}$ determined by the root $\alpha_{i}$. If $w=s_{i_{1}} s_{i_{2}} \ldots s_{i_{m}}$ is the shortest expansion of $w$ in $s_{i}$ 's, then $s_{i_{1}} s_{i_{2}} \ldots s_{i_{m}}$ is called a reduced expression for 
$w$ and $\ell(w):=m$ is the length of $w$. The Bruhat ordering on $W$ is the partial ordering generated by the relations $w_{1}<w_{2}$ satisfying $s_{\alpha} w_{1}=w_{2}$ and $\ell\left(w_{1}\right)+1=\ell\left(w_{2}\right)$ for some positive root $\alpha \in \Lambda_{+}$. It is known that there is a unique maximal element in $W$ with respect to the Bruhat ordering.

Soibelman's classification of irreducible *-representations of $C\left(K_{q}\right)^{\infty}$ (or of $C\left(K_{q}\right)$ ) can be summarized by the following.

(1) One-dimensional irreducible *-representations $\tau_{t}$ of $C\left(K_{q}\right)^{\infty}$ are parametrized by $t \in \mathbb{T}^{r}$, the maximal torus in $K$.

(2) Irreducible *-representations of $C\left(K_{q}\right)^{\infty}$ are parametrized by elements $(t, w)$ of $\mathbb{T}^{r} \times W$. In fact, if $t \in \mathbb{T}^{r}$ and $w=s_{i_{1}} s_{i_{2}} \ldots s_{i_{m}}$ is a reduced expression for $w$, then $\left(\tau_{t} \otimes \pi_{i_{1}} \otimes \pi_{i_{2}} \otimes \ldots \otimes \pi_{i_{m}}\right) \Delta^{m}$ is the corresponding irreducible ${ }^{*}$-representation (independent of the choice of the reduced expression for $w$ ), where $\Delta$ is the comultiplication on $C\left(K_{q}\right)^{\infty}$ and $\Delta^{m}$ is defined recursively as $\Delta^{k}=(\Delta \bigotimes i d) \Delta^{k-1}$.

It is an interesting discovery [Sol] that the symplectic leaves $L$ in $K$ are in oneto-one correspondence with elements $(t, w)$ of $\mathbb{T}^{r} \times W$ and hence with the irreducible *-representations $\pi_{L}$ of $C\left(K_{q}\right)^{\infty}$. Indeed if $t \in \mathbb{T}^{r}$ and $w=s_{i_{1}} s_{i_{2}} \ldots s_{i_{m}}$ is a reduced expression for $w$, then the set $t S_{i_{1}} S_{i_{2}} \ldots S_{i_{m}} \subset K$ is the corresponding symplectic leaf, where $S_{i}=\phi_{i_{*}}(S)$ with

$$
S=\left\{\left(\begin{array}{cc}
\alpha & -\gamma \\
\gamma & \bar{\alpha}
\end{array}\right): \alpha \in \mathbb{C},|\alpha|<1, \gamma=\sqrt{1-|\alpha|^{2}}\right\}
$$

the prominent 2-dimensional symplectic leaf in $S U(2)$. Completing $C\left(K_{q}\right)^{\infty}$ with respect to the norm $\|x\|:=\sup _{L}\left\|\pi_{L}(x)\right\|$, we get the type I C*-algebra $C\left(K_{q}\right)$ [So1].

From the above result, we can talk about symplectic leaf-preserving quantizations of $K$ by $K_{q}$ and group- (or comultiplication-) preserving quantizations of $K$ by $K_{q}$. It is interesting to know that there is no quantization of $K$ by $K_{q}$ which is simultaneously leaf-preserving and group-preserving [Sh3, Sh4]. On the other hand, surprisingly, Rieffel showed that for ${ }_{u} K_{q}$ with $u \neq 0$, there does exist such a quantization [Ri4].

3. Groupoids for $K_{q}$. It has been well recognized that groupoid $\mathrm{C}^{*}$-algebras provide a very powerful tool to study the structure of concrete $\mathrm{C}^{*}$-algebras like Toeplitz $\mathrm{C}^{*}$ algebras, Wiener-Hopf $\mathrm{C}^{*}$-algebras, etc. For the theory of groupoid $\mathrm{C}^{*}$-algebras, we refer to Renault's book [Re].

Recall that the transformation group groupoid $\mathbb{Z}^{m} \times \overline{\mathbb{Z}}^{m}$ (with $\mathbb{Z}^{m}$ acting on $\overline{\mathbb{Z}}^{m}$ by translation) when restricted to the positive cone $\overline{\mathbb{Z}}_{\geq}^{m}$ gives an important (Toeplitz) groupoid

$$
\mathbb{Z}^{m} \times\left.\overline{\mathbb{Z}}^{m}\right|_{\overline{\mathbb{Z}}_{\geq}^{m}}:=\left\{(j, k) \in \mathbb{Z}^{m} \times \overline{\mathbb{Z}}_{\geq}^{m} \mid j+k \in \overline{\mathbb{Z}}_{\geq}^{m}\right\}
$$

where $\overline{\mathbb{Z}}=\mathbb{Z} \cup\{+\infty\}$ and $\bar{Z}_{\geq}:=\{0,1,2,3, \ldots\} \cup\{+\infty\}$.

Let $s_{i_{1}} s_{i_{2}} \ldots s_{i_{N}}$ be a reduced expression for the unique maximal element in the Weyl group with respect to the Bruhat ordering. Then all irreducible *-representations of $C\left(K_{q}\right)$ factor through the $\mathbb{T}^{r}$-family $\left(\tau_{t} \otimes \pi_{i_{1}} \otimes \pi_{i_{2}} \otimes \ldots \otimes \pi_{i_{N}}\right) \Delta^{N}$ of representations. The $\mathbb{T}^{r}$-family $\left\{\tau_{t}\right\}_{t \in \mathbb{T}^{r}}$ of one-dimensional irreducible ${ }^{*}$-representations of $C\left(K_{q}\right)$ can be viewed as a $\mathrm{C}^{*}$-algebra homomorphism $\tau: C\left(K_{q}\right) \rightarrow C\left(\mathbb{T}^{r}\right) \cong C^{*}\left(\mathbb{Z}^{r}\right)$. Now it is 
clear that all irreducible ${ }^{*}$-representations of $C\left(K_{q}\right)$ factor through the homomorphism $\left(\tau \otimes \pi_{i_{1}} \otimes \pi_{i_{2}} \otimes \ldots \otimes \pi_{i_{N}}\right) \Delta^{N}$. Thus we get the following theorem [Sh5].

Theorem 1. $C\left(K_{q}\right)$ can be embedded into

$$
C^{*}\left(\mathbb{Z}^{r} \times \mathbb{Z}^{N} \times\left.\overline{\mathbb{Z}}^{N}\right|_{\overline{\mathbb{Z}}_{\geq}^{N}} \subseteq C\left(\mathbb{T}^{r}\right) \otimes \mathcal{B}\left(\ell^{2}\left(\mathbb{Z}_{\geq}^{N}\right)\right)\right.
$$

by $\left(\tau \otimes \pi_{i_{1}} \otimes \pi_{i_{2}} \otimes \ldots \otimes \pi_{i_{N}}\right) \Delta^{N}$, where $\mathbb{Z}^{r}$ acts trivially on $\overline{\mathbb{Z}}^{N}$ and $\mathbb{Z}^{N}$ acts by translation on $\overline{\mathbb{Z}}^{N}$.

Let us consider the special case of $G=S L(n+1)$ and $K=S U(n+1)$ with $n \geq 1$, for which $r=n$. The $\mathrm{C}^{*}$-algebra $C\left(S U(n+1)_{q}\right)$ is generated by $u_{i j}, 1 \leq i, j \leq n+1$, satisfying $u^{*} u=u u^{*}=I$ and some other relations [Wo3, So1].

Irreducible 1-dimensional *-representations of $C\left(S U(n+1)_{q}\right)$ are defined by $\tau_{t}\left(u_{i j}\right)=$ $\delta_{i j} t_{j}$ for $t \in \mathbb{T}^{n}$ (with $\left.t_{n+1}=t_{1}^{-1} t_{2}^{-1} \ldots t_{n}^{-1}\right)$, and we set $\tau_{n+1}=\tau: C\left(S U(n+1)_{q}\right) \rightarrow$ $C^{*}\left(\mathbb{Z}^{n}\right)$. There are $n$ fundamental *-representations $\pi_{i}=\pi_{0} \phi_{i}$ with $\phi_{i}: C\left(S U(n+1)_{q}\right) \rightarrow$ $C\left(S U(2)_{q}\right)$ given by $\phi_{i}\left(u_{j k}\right)=u_{j-i+1, k-i+1}$ if $\{j, k\} \subseteq\{i, i+1\}$ and $\phi_{i}\left(u_{j k}\right)=\delta_{j k}$ if otherwise.

The unique maximal element in the Weyl group of $S U(n+1)$ can be expressed in the reduced form

$$
s_{1} s_{2} s_{1} s_{3} s_{2} s_{1} \ldots s_{n} s_{n-1} \ldots s_{2} s_{1} .
$$

So $C\left(S U(n+1)_{q}\right)$ can be embedded into

$$
C^{*}\left(\mathcal{G}^{n}\right) \subseteq C^{*}\left(\mathbb{Z}^{n}\right) \otimes \mathcal{B}\left(\ell^{2}\left(\mathbb{Z}_{\geq}^{N}\right)\right)
$$

by

$$
\left(\tau_{n+1} \otimes \pi_{121321 \ldots n(n-1) . .21}\right) \Delta^{N}
$$

where $N=n(n+1) / 2$,

$$
\pi_{i_{1} i_{2} \ldots i_{m}}:=\pi_{i_{1}} \otimes \pi_{i_{2}} \otimes \ldots \otimes \pi_{i_{m}},
$$

and $\mathcal{G}^{n}$ is the groupoid $\mathbb{Z}^{n} \times \mathbb{Z}^{N} \times\left.\overline{\mathbb{Z}}^{N}\right|_{\overline{\mathbb{Z}}_{>}^{N}}$ with $\mathbb{Z}^{n}$ acting trivially on $\overline{\mathbb{Z}}^{N}$, and $\mathbb{Z}^{N}$ acts by translation on $\overline{\mathbb{Z}}^{N}$.

With a minor modification, we can study the related quantum $U(n)_{q}$ in a similar way.

4. Structure theorems for $C\left(S U(n)_{q}\right)$. Applying the above groupoid approach to $S U(n)_{q}$, we get the following structure theorems for $C\left(S U(n)_{q}\right)$ [Sh5].

For any subset $J$ of $\{1,2, \ldots, N\}$, we define

$$
X_{J}:=\left\{k \in \overline{\mathbb{Z}}_{\geq}^{N} \mid k_{i}=\infty \text { if } i \notin J\right\}
$$

an invariant closed subset of the unit space of $\mathcal{G}^{n}$, called a face of $\overline{\mathbb{Z}}_{\geq}^{N}$. By restricting the embedded algebra $C\left(S U(n+1)_{q}\right)$ to various faces $X_{J}$, we can analyze its algebra structure and get interesting composition sequences of $C\left(S U(n+1)_{q}\right)$.

$C\left(S U(n+1)_{q}\right)$ is determined by $\left.C\left(S U(n+1)_{q}\right)\right|_{X_{J}}$ with admissible $J$ only. $(J$ is

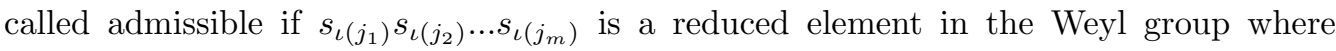
$\iota:\{1,2, \ldots, N\} \rightarrow\{1,2, \ldots, n\}$ is defined by

$$
(\iota(1), \ldots, \iota(N))=(1,2,1,3,2,1, \ldots, n, n-1 \ldots, 1) .)
$$


In fact, $J$ is admissible if and only if for each $0 \leq k<n$, there is some $0 \leq m_{k} \leq k+1$ such that

$$
J=\left\{j \mid \quad\left(\frac{k^{2}+k}{2}\right)<j \leq\left(\frac{k^{2}+k}{2}\right)+m_{k}, \quad \text { for some } 0 \leq k<n\right\} .
$$

Let $\mathcal{L}_{k}$ be the collection of admissible $J \subseteq J_{n}$ with size $|J|=k$, for $0 \leq k \leq N$, and set $X_{k}=\cup_{J \in \mathcal{L}_{k}} X_{J}$.

Theorem 2. The $C^{*}$-algebra $C\left(S U(n+1)_{q}\right)$ has the composition sequence

$$
C\left(S U(n+1)_{q}\right)=\mathcal{I}_{0} \supseteq \mathcal{I}_{1} \supseteq \ldots \supseteq \mathcal{I}_{N} \supseteq \mathcal{I}_{N+1}:=0,
$$

with

$$
\mathcal{I}_{k} / \mathcal{I}_{k+1} \simeq \bigoplus_{J \in \mathcal{L}_{k}} C\left(\mathbb{T}^{n}\right) \otimes \mathcal{K}\left(\ell^{2}\left(\mathbb{Z}_{\geq}^{k}\right)\right)
$$

where $\mathcal{I}_{k}$ are ideals of $C\left(S U(n+1)_{q}\right)$ such that

$$
\left.C\left(S U(n+1)_{q}\right)\right|_{X_{N-k}} \simeq C\left(S U(n+1)_{q}\right) / \mathcal{I}_{k} .
$$

$\left(\right.$ Here we use $\mathcal{K}\left(\ell^{2}\left(\mathbb{Z}_{\geq}^{0}\right)\right)=\mathbb{C}$. $)$

Remark. Each $C\left(\mathbb{T}^{n}\right) \otimes \mathcal{K}\left(\ell^{2}\left(\mathbb{Z}_{>}^{k}\right)\right)$ corresponds to a $\mathbb{T}^{n}$-family of $2 k$-dimensional symplectic leaves.

Theorem 3. For $C\left(S U(n)_{q, m}\right):=\left.C\left(S U(n+1)_{q}\right)\right|_{X_{J}}$ with $J=\{1,2, \ldots, N-m+1\}$, there are short exact sequences

$$
\begin{gathered}
0 \rightarrow \mathcal{A} \otimes \mathcal{K} \rightarrow C\left(S U(n)_{q, n}\right) \rightarrow C\left(S U(n)_{q, n+1}\right) \rightarrow 0 \\
0 \rightarrow \mathcal{A} \otimes \mathcal{K} \rightarrow C\left(S U(n)_{q, n-1}\right) \rightarrow C\left(S U(n)_{q, n}\right) \rightarrow 0 \\
\ldots \\
0 \rightarrow \mathcal{A} \otimes \mathcal{K} \rightarrow C\left(S U(n)_{q, 1}\right) \rightarrow C\left(S U(n)_{q, 2}\right) \rightarrow 0
\end{gathered}
$$

with

$$
\mathcal{A}=C(\mathbb{T}) \bigotimes C\left(S U(n)_{q}\right) \simeq C\left(S U(n+1)_{q, n+1}\right)
$$

and $C\left(S U(n)_{q, 1}\right)=C\left(S U(n+1)_{q}\right)$. Furthermore, there is an element

$$
1 \otimes T \in C\left(S U(n)_{q, m}\right) \subseteq C\left(S U(n)_{q, n+1}\right) \otimes \mathcal{B}\left(\ell^{2}\left(\mathbb{Z}_{\geq}^{n-m+1}\right)\right)
$$

such that $T$ is a Fredholm operator with index 1.

These short exact sequences correspond to the classical fibration of $S U(n+1)$ over $\mathbb{C} P(n)$ by fibres $U(n)$.

Corollary 4. The $C^{*}$-algebra $C\left(S U(n+1)_{q}\right)$ has the composition sequence

$$
C\left(S U(n+1)_{q}\right)=\mathcal{I}_{0} \supseteq \mathcal{I}_{1} \supseteq \ldots \supseteq \mathcal{I}_{n} \supseteq \mathcal{I}_{n+1}:=0,
$$

with

$$
\mathcal{I}_{k} / \mathcal{I}_{k+1} \simeq C\left(U(n)_{q}\right) \otimes \mathcal{K}\left(\ell^{2}\left(\mathbb{Z}_{\geq}^{k}\right)\right)
$$

for $k>0$ and $\mathcal{I}_{0} / \mathcal{I}_{1} \simeq C\left(U(n)_{q}\right) \cong C(T) \otimes C\left(S U(n)_{q}\right)$. 
Corollary 5. The $C^{*}$-algebra $C\left(S U(n+1)_{q}\right)$ has the composition sequence

$$
C\left(S U(n+1)_{q}\right)=\mathcal{I}_{0} \supseteq \mathcal{I}_{1} \supseteq \ldots \supseteq \mathcal{I}_{(n+1) !}:=0,
$$

with

$$
\mathcal{I}_{k} / \mathcal{I}_{k+1} \simeq C\left(\mathbb{T}^{n}\right) \otimes \mathcal{K}
$$

for $k>0$ and $\mathcal{I}_{0} / \mathcal{I}_{1} \simeq C\left(\mathbb{T}^{n}\right)$

5. Quantum spheres. Similar structure theorems have also been obtained for quantum spheres by using this groupoid approach [Sh5]. by

Recall that Nagy [N] considered quantum homogeneous spaces $M_{q}=H_{q} \backslash K_{q}$ defined

$$
C\left(M_{q}\right)=\left\{f \in C\left(K_{q}\right):(\Phi \otimes i d)(\Delta f)=1 \otimes f\right\}
$$

where $H$ is a closed subgroup of $K$ and $\Phi: C\left(K_{q}\right) \rightarrow C\left(H_{q}\right)$ is the quantization of the embedding homomorphism from $H$ into $K$. With $(K, H)=(S U(n+1), S U(n))$, we get (odd-dimensional) quantum spheres $S_{q}^{2 n+1}=S U(n)_{q} \backslash S U(n+1)_{q}$.

Proposition 6.

$$
C\left(S_{q}^{2 n+1}\right)=C^{*}\left(\left\{u_{n+1, m} \mid 1 \leq m \leq n+1\right\}\right)
$$

and $\tau_{n+1} \otimes \pi_{n} \otimes \pi_{n-1} \otimes \ldots \otimes \pi_{1}$ gives an embedding of $C\left(S_{q}^{2 n+1}\right)$ in $C^{*}\left(\mathcal{F}^{n}\right)$, where $\mathcal{F}^{n}=\mathbb{Z} \times\left(\mathbb{Z}^{n} \times\left.\overline{\mathbb{Z}}^{n}\right|_{\overline{\mathbb{Z}}_{\geq}^{n}}\right)$ and $\tau_{n+1}\left(u_{n+1, m}\right)=\left(\delta_{n+1, m}\right) \delta_{1} \in C^{*}(\mathbb{Z})$.

Proposition 7. There is a short exact sequence

$$
0 \rightarrow C(\mathbb{T}) \otimes \mathcal{K} \rightarrow C\left(S_{q}^{2 k+1}\right) \rightarrow C\left(S_{q}^{2 k-1}\right) \rightarrow 0
$$

for $k \geq 1$ with $C\left(S_{q}^{1}\right) \simeq C(\mathbb{T})$ such that $C\left(S_{q}^{2 k+1}\right)$ contains an element $1 \otimes T \in C(\mathbb{T}) \otimes$ $\mathcal{B}\left(\ell^{2}\left(\mathbb{Z}_{\geq}^{k}\right)\right)$ where $T$ is a Fredholm operator with index 1 .

COROLlaRY 8. The $C^{*}$-algebra $C\left(S_{q}^{2 n+1}\right)$ has the composition sequence

$$
C\left(S_{q}^{2 n+1}\right)=\mathcal{I}_{0} \supseteq \mathcal{I}_{1} \supseteq \ldots \supseteq \mathcal{I}_{n} \supseteq \mathcal{I}_{n+1}:=0,
$$

with

$$
\mathcal{I}_{k} / \mathcal{I}_{k+1} \simeq C(\mathbb{T}) \otimes \mathcal{K}\left(\ell^{2}\left(\mathbb{Z}^{k}\right)\right)
$$

for $k>0$ and $\mathcal{I}_{0} / \mathcal{I}_{1} \simeq C(\mathbb{T})$.

6. Haar functional. In [Wo2], Woronowicz proved the existence of the important invariant Haar functional on compact matrix quantum groups. Using the groupoid approach, we can give an explicit description of the Haar functional $h_{n}$ on $C\left(S U(n)_{q}\right)$ [Sh5].

THEOREM 9. The unique invariant functional $h_{n+1}$ on $C\left(S U(n+1)_{q}\right)$ is the restriction of the state $f_{\xi^{(n)}}$ of $C^{*}\left(\mathcal{G}^{n}\right)$ given by the regular representation on $\ell^{2}\left(\left.\mathcal{G}^{n}\right|_{\mathbb{Z}_{>}^{N}}\right)$ and

$$
\xi^{(n)}=\left(\prod_{i=1}^{n}\left(1-q^{-2(n+1-i)}\right)^{-i / 2}\right)
$$




$$
\sum_{w \in \mathbb{Z}_{\geq}^{N}} q^{-\Sigma(n+1-[i]) w_{i}} \cdot \delta_{(0,0, w)} \in \ell^{2}\left(\left.\mathcal{G}^{n}\right|_{\mathbb{Z}_{\geq}^{N}}\right),
$$

i.e. $h_{n+1}=f_{\xi^{(n)}} \circ\left(\tau_{n+1} \otimes \pi_{J_{n}}\right)$, where $[i] \geq 0$ is defined by $i=[i]+k(k+1) / 2$ and $[i] \leq k+1$.

This result can be used to prove the known facts that $[\mathrm{N}] h_{n+1}$ is a faithful state on $C\left(S U(n+1)_{q}\right)$, and that $K_{i}\left(C\left(S_{q}^{2 n+1}\right)\right) \simeq Z$ for both $i=0$ and 1 [VSo2].

7. Subquotient groupoids. It is an interesting question whether $C\left(S_{q}^{2 n+1}\right)$ (or $\left.C\left(S U(n)_{q}\right)\right)$ is really a groupoid $\mathrm{C}^{*}$-algebra of some groupoid instead of a $\mathrm{C}^{*}$-subalgebra of some groupoid $\mathrm{C}^{*}$-algebra.

It is not difficult to see that the answer is affirmative for the following two simple but fundamental examples.

(1) $C\left(S U(2)_{q}\right) \simeq C^{*}\left(\mathfrak{G}_{1}\right)$ where

$$
\mathfrak{G}_{1}=\{(\mathfrak{m}, \mathfrak{j}, \mathfrak{k}) \mid \text { if } \mathfrak{k}=\infty \text {, then } \mathfrak{m}=\mathfrak{o}\}
$$

is a subgroupoid of the groupoid $\mathbb{Z} \times\left(\mathbb{Z} \times\left.\overline{\mathbb{Z}}\right|_{\overline{\mathbb{Z}}_{>}}\right)$, with

$$
u_{11}=\sum_{0<j \leq \infty}\left(1-q^{-2 j}\right)^{1 / 2} \delta_{(0,-1, j)}
$$

and $u_{21}=\sum_{0 \leq j<\infty} q^{-j} \delta_{(1,0, j)}$.

(2) Podles' quantum sphere $C\left(S_{\mu c}^{2}\right) \simeq C^{*}\left(\mathfrak{G}^{\prime}\right), c>0$, where

$$
\mathfrak{G}^{\prime}=\left\{\left(\mathfrak{j}, \mathfrak{j}, \mathfrak{k}_{1}, \mathfrak{k}_{2}\right) \mid \mathfrak{k}_{1}=\infty \text { or } \mathfrak{k}_{2}=\infty\right\}
$$

is a subgroupoid of the groupoid $\mathbb{Z}^{2} \times\left.\overline{\mathbb{Z}}^{2}\right|_{\overline{\mathbb{Z}}_{>}^{2}}$.

It turns out that the answer is also affirmative for odd-dimensional quantum spheres $S_{q}^{2 n+1}$ and for quantum $S U(3)_{q}$.

Define a subquotient groupoid $\mathfrak{F}_{\mathfrak{n}}$ of $\mathcal{F}^{n}=\mathbb{Z} \times\left(\mathbb{Z}^{n} \times\left.\overline{\mathbb{Z}}^{n}\right|_{\overline{\mathbb{Z}}_{>}^{n}}\right)$ as follows. Let

$$
\begin{gathered}
\widetilde{\mathfrak{F}_{\mathfrak{n}}}:=\left\{(m, j, k) \in \mathcal{F}^{n} \mid k_{i}=\infty \Longrightarrow\right. \\
\left.j_{i}=m-j_{1}-j_{2}-\ldots-j_{i-1} \text { and } j_{i+1}=\ldots=j_{n}=0\right\}
\end{gathered}
$$

be a subgroupoid of $\mathcal{F}^{n}$. Define $\mathfrak{F}_{\mathfrak{n}}:=\widetilde{\mathfrak{F}_{\mathfrak{n}}} / \sim$ where $\sim$ is the equivalence relation generated by

$$
(m, j, k) \sim\left(m, j, k_{1}, \ldots, k_{i}=\infty, \infty, \ldots, \infty\right)
$$

for all $(m, j, k)$ with $k_{i}=\infty$ for an $1 \leq i \leq n$.

THEOREM 10. $C\left(S_{q}^{2 n+1}\right) \simeq C^{*}\left(\mathfrak{F}_{\mathfrak{n}}\right)$.

From this, we get the following known result [VSo2].

Corollary 11. The $C^{*}$-algebra $C\left(S_{q}^{2 n+1}\right)$ is independent of $q$.

The key technical point in proving that $C\left(S U(3)_{q}\right)$ is a groupoid $\mathrm{C}^{*}$-algebra is based on Lance's [La] (or Woronowicz's [Wo4]) result that there exists some isometry

$$
v: \ell^{2}(\mathbb{Z} \times \mathbb{N} \times \mathbb{Z} \times \mathbb{N}) \rightarrow \ell^{2}(\mathbb{Z} \times \mathbb{N} \times \mathbb{Z} \times \mathbb{Z})
$$


such that

$$
v(\Delta x) v^{*}=x \otimes 1
$$

for $x \in C\left(S U(2)_{q}\right) \subset \mathcal{B}\left(\ell^{2}\left(\mathbb{Z} \times \mathbb{Z}_{\geq}\right)\right)$. Modifying this result, we can show that

Proposition 12. There is some isometry

$$
w: \ell^{2}\left(\mathbb{Z}_{\geq} \times \mathbb{Z}_{\geq}\right) \rightarrow \ell^{2}(\mathbb{Z} \times \mathbb{Z})
$$

such that

$$
w\left(\left(\pi_{1} \otimes \pi_{1}\right) \Delta(x)\right) w^{*}=\left(\tau \otimes \pi_{1}\right) \Delta(x)
$$

for $x \in C\left(S U(3)_{q}\right)$.

As far as the algebra $C\left(S U(3)_{q}\right)$ is concerned, the face $X_{\{1,3\}}$ corresponding to $\pi_{1} \otimes \pi_{1}$ is 'degenerate' and is 'dominated' by the face $X_{\{1,2\}}$ corresponding to $\pi_{1} \otimes \pi_{2}$. This combined with the above composition sequence for $C\left(S U(3)_{q}\right)$ helps to build a quotient groupoid $\mathfrak{G}_{2}$ from a subgroupoid $\widetilde{\mathfrak{G}}_{2}$ of $\mathcal{G}^{2}=\mathbb{Z}^{2} \times\left(Z^{3} \times\left.\overline{\mathbb{Z}}^{3}\right|_{\overline{\mathbb{Z}}_{>}^{3}}\right)$ where

$$
\begin{aligned}
\widetilde{\mathfrak{G}}_{2}:=\{ & (m, j, k) \in \mathcal{G}^{2} \mid m_{1}+m_{2}+m_{3}=0, \\
& k_{1}=\infty \Longrightarrow j_{1}=m_{1}-m_{2}, \\
& k_{2}=\infty \Longrightarrow j_{2}=m_{1}-m_{3}-j_{1}, \\
& \left.k_{3}=\infty \Longrightarrow j_{3}=m_{2}-m_{3}+j_{1}-j_{2}\right\} .
\end{aligned}
$$

We use the following notations: $r \wedge s:=\min \{r, s\}, r \vee s:=\max \{r, s\}$,

$$
\psi(r, s):=(r-s) \vee 0-(s-r) \vee 0,
$$

and $\eta(r):=(r, 0)$ if $r>0$ and $\eta(r):=(0,-r)$ if $r<0$. Define $\mathfrak{G}_{2}:=\widetilde{\mathfrak{G}}_{2} / \sim$, the quotient groupoid given by the equivalence relation $\sim$ generated by

$$
\begin{aligned}
& \left(m, j_{1}, j_{2}, j_{3}, k_{1}, k_{2}=\infty, k_{3}\right) \sim \\
& \quad\left(m, \tilde{j}_{1}, j_{2}+j_{1}-\tilde{j}_{1}, \tilde{j}_{3}, k_{1} \wedge k_{3}, k_{2}=\infty, k_{1} \wedge k_{3}\right)
\end{aligned}
$$

where

$$
\begin{aligned}
\left(\tilde{j}_{1}, \tilde{j}_{3}\right): & =\left(\left(k_{1}+l_{1}\right) \wedge\left(k_{3}+l_{3}\right)-k_{1} \wedge k_{3}\right)(1,1) \\
& +\eta\left(\psi\left(k_{1}+l_{1}, k_{3}+l_{3}\right)-\psi\left(k_{1}, k_{3}\right)\right) .
\end{aligned}
$$

THEOREM 13. $C\left(S U(3)_{q}\right) \cong C^{*}\left(\mathfrak{G}_{2}\right)$.

From this, we get a result of Nagy.

Corollary 14. $C\left(S U(3)_{q}\right)$ as a $C^{*}$-algebra is independent of $q$.

It is interesting to note that $\mathrm{S}$. Wang [Wa] has proved that for $n \geq 2, C\left(S U(n)_{q}\right)$ with different $q$ 's are not isomorphic as Hopf $\mathrm{C}^{*}$-algebras.

\section{References}

[B-D] A. Belavin and V. Drinfeld, Solutions of the classical Yang-Baxter equation for simple Lie algebras, Func. Anal. Appl. 16 (1982).

[Co] A. Connes, A survey of foliation and operator algebras, Proc. Symp. Pure Math. Vol. 38, Part I, AMS, Providence, 1982, 521-628. 
[CuM] R. E. Curto and P. S. Muhly, $C^{*}$-algebras of multiplication operators on Bergman spaces, J. Func. Anal. 64 (1985), 315-329.

[D] V. G. Drinfeld, Quantum groups, Proc. I.C.M. Berkeley 1986, Vol. 1, 789-820, Amer. Math. Soc., Providence, 1987.

[H] H. Hiller, Geometry of Coxeter Groups, Research Notes in Math. Vol. 54, Pitman, Boston, 1982.

[La] C. Lance, An explicit description of the fundamental unitary for $S U(2)_{q}$, Comm. Math. Phys. 164 (1994), 1-15.

[LeSo] S. Levendorskii and Ya. Soibelman, Algebras of functions on compact quantum groups, Schubert cells and quantum tori, Comm. Math. Phys., 139 (1991), 141-170.

[LuWe] J. H. Lu and A. Weinstein, Poisson Lie groups, dressing transformations and Bruhat decompositions, J. Diff. Geom. 31 (1990), 501-526.

[MRe] P. S. Muhly and J. N. Renault, $C^{*}$-algebras of multivariable Wiener-Hopf operators, Trans. Amer. Math. Soc. 274 (1982), 1-44.

[N] G. Nagy, On the Haar measure of the quantum $S U(N)$ group, Comm. Math. Phys. 153 (1993), 217-228.

[Po] P. Podleś, Quantum spheres, Letters Math. Phys. 14 (1987), 193-202.

[Re] J. Renault, A Groupoid Approach to $\mathrm{C}^{*}$-algebras, Lecture Notes in Mathematics, Vol. 793, Springer-Verlag, New York, 1980.

[RTF] N. Yu. Reshetikhin, L. A. Takhtadzhyan, and L. D. Faddeev, Quantization of Lie groups and Lie algebras, Leningrad Math. J. 1 (1990), 193-225.

[Ri1] M. A. Rieffel, Deformation quantization and operator algebras, in "Proc. Symp.Pure Math., Vol. 51", AMS, Providence, 1990, pp. 411-423.

[Ri2] - Deformation quantization for actions of $\mathbb{R}^{d}$, Memoirs of AMS, Vol. 106, No. 506, 1993.

[Ri3] -, Compact quantum groups associated with toral subgroups, in "Contemporary Mathematics", Vol. 145, AMS, Providence, 1993, pp. 465-491.

[Ri4] -, Non-compact quantum groups associated with abelian subgroups, Comm. Math. Phys., 171 (1995), 181-201.

[Sh1] A. J. L. Sheu, Reinhardt domains, boundary geometry and Toeplitz $C^{*}$-algebras, Journal of Functional Analysis, 92 (1990), 264-311.

[Sh2] - Quantization of the Poisson SU(2) and its Poisson homogeneous space - the 2-sphere, Comm. Math. Phys. 135 (1991), 217-232.

[Sh3] - Leaf-preserving quantizations of Poisson SU(2) are not coalgebra homomorphisms, Comm. Math. Phys., 172 (1995), 287-292.

[Sh4] —, Symplectic leaves and deformation quantization, Proc. Amer. Math. Soc., 124 (1996), 95-100.

[Sh5] - Compact quantum groups and groupoid $C^{*}$-algebras, to appear in J. Func. Anal.

[So1] Ya. S. Soibelman, The algebra of functions on a compact quantum group, and its representations, Algebra Analiz. 2 (1990), 190-221. (Leningrad Math. J., 2 (1991), 161-178.)

[So2] - Irreducible representations of the function algebra on the quantum group $S U(n)$, and Schubert cells, Soviet Math. Dokl. 40 (1990), 34-38.

[VSo1] L. L. Vaksman and Ya. S. Soibelman, Algebra of functions on the quantum group $S U(2)$, Func. Anal. Appl. 22 (1988), 170-181.

[VSo2] - , - The algebra of functions on the quantum group $S U(n+1)$, and odd-dimensional quantum spheres, Leningrad Math. J. 2 (1991), 1023-1042.

[Wa] S. Wang, Classification of quantum groups $S U_{q}(n)$, preprint. 
[We] A. Weinstein, The local structure of Poisson manifolds, J. Diff. Geom. 18 (1983), 523557.

[Wo1] S. L. Woronowicz, Twisted SU(2) group: an example of a non-commutative differential calculus, Publ. RIMS. 23 (1987), 117-181.

[Wo2] —, Compact matrix pseudogroups, Comm. Math. Phys. 111 (1987), 613-665.

[Wo3] -, Tannaka-Krein duality for compact matrix pseudogroups, twisted $S U(N)$ groups, Invent. Math. 93 (1988), 35-76.

[Wo4] -, Quantum SU (2) and E (2) groups. Contraction procedure, Comm. Math. Phys. 149 (1992), 637-652. 